\title{
Growth of Aspergillus and Mucor spp. in sputum culture of a post-COVID patient: fungal infection or colonization? A clinical challenge in COVID-era
} Chowdhury TA ${ }^{a}$, Haque TSU ${ }^{b}$, Barai L ${ }^{c}$, Rahim MA ${ }^{d}$, Ananna MAd, Jahan I ${ }^{e}$, Iqbal $S^{f}$

\begin{abstract}
Opportunistic infections, especially invasive fungal infections have emerged as an added health issue in this COVID-era. The dysregulated immune mechanisms in the pathophysiology of COVID-19, its treatment with corticosteroids and other immunomodulatory agents, invasive mechanical ventilation and other forms of ventilatory and oxygen delivery systems, prolonged hospital stay, injudicious use of antimicrobials - all have set the required stages for such infections. Aspergillus spp. and Mucorales are ubiquitous, environmental fungus; may colonize in airways and chronically damaged human lungs, without significant health effects and cause disease, especially in immunocompromized patients. We report case history of a middle aged Bangladeshi man with multiple comorbidities, who presented with fever and respiratory symptoms, four-months after recovery from COVID-19. He had Aspergillus and Mucors on sputum microscopy and cultures; but his response to antibiotics suggested these were colonozations in previously injured lungs by tuberculosis in the form of fibrosis and bronchiectasis. He is well and free of symptoms, two-months since discharge.
\end{abstract}

Key words: colonization, COVID-19, invasive fungal infection, pulmonary aspergillosis, pulmonary mucormycosis.

(BIRDEM Med J 2021; 11(3): 218-222)

\section{INTRODUCTION}

Invasive fungal infections of the respiratory tract are uncommon and generally occur in patients with immunosuppression. Predominant agents responsible for invasive respiratory infections are Aspergillus spp.

\section{Author information}

a. Tufayel Ahmed Chowdhury, Assistant Professor, Department of Nephrology, BIRDEM General Hospital, Dhaka, Bangladesh.

b. Talha-Sami-Ul-Haque, Resident Medical Officer, Department of Nephrology, BIRDEM General Hospital, Dhaka, Bangladesh.

c. Lovely Barai, Associate Professor, Department of Microbiology, BIRDEM General Hospital, Dhaka, Bangladesh.

d. Muhammad Abdur Rahim, Mehruba Alam Ananna, Associate Professor, Department of Nephrology, BIRDEM General Hospital, Dhaka, Bangladesh.

e. Israt Jahan, Assistant Registrar, Department of Nephrology, BIRDEM General Hospital, Dhaka, Bangladesh.

f. Sarwar Iqbal, Professor, Department of Nephrology, BIRDEM General Hospital, Dhaka, Bangladesh.

Address of correspondence: Tufayel Ahmed Chowdhury, Assistant Professor, Department of Nephrology, BIRDEM General Hospital, Dhaka, Bangladesh. Email: dr_topu11@yahoo.com

Received: July 12, 2021

Revision received: July 30, 2021

Accepted: July 31, 2021 and Mucorales. ${ }^{1}$ These ubiquitous agents may colonize in previously damaged lungs like cavities of tuberculosis, lung cancer etc. even without noticeable symptoms to the host. ${ }^{2,3}$ It is important to differentiate pulmonary fungal colonization from invasive fungal infections; as the former may require careful follow up only but the later becomes fatal without definite treatment. Here, we present a case of pulmonary fungal colonizations.

\section{CASE REPORT}

A 49-year-old Bangladeshi male patient from Shibchar of Madaripur district, presented with a 15-day history of fever, cough and chest pain. He is a known case of diabetes mellitus, hypertension, chronic kidney disease (stage 5) and hepatitis B virus associated chronic liver disease. His past medical history included smear positive pulmonary tuberculosis in 2007 and 2017 (treated according to the National Guidelines on both occasions) and moderate COVID-19 in December 2020. His inhospital treatment for COVID-19 included dexamethasone $6 \mathrm{mg}$ /day for 5 days and oxygen supplement as on required basis.

His fever was intermittent, occasionally reached up to $103^{\circ} \mathrm{F}$. His cough was productive in nature, more on 
supine position but he denied any shortness of breath or haemoptysis. He complained of left-sided chest pain during cough. Three days prior to hospitalization, he developed runny nose. He took paracetamol and antihistamines without much benefit.

Evaluation at our center during admission revealed, his respiratory rate was 16 breaths $/ \mathrm{min}$, pulse 90 beats $/ \mathrm{min}$ and blood pressure 160/80 $\mathrm{mm} \mathrm{Hg}$. His oxygen saturation was $96 \%$ while breathing ambient air. Chest auscultation revealed bilateral crepitations and diminished breath sound on the left lower chest. Other systemic examination findings were unremarkable.

Laboratory reports showed anaemia (haemoglobin 9.2 $\mathrm{gm} / \mathrm{dl}$ ), leukocytosis (total white cells $11840 / \mathrm{cmm}$ ), high erythrocyte sedimentation rate (ESR) $(100 \mathrm{~mm}$ in $1 \mathrm{st}$ hour) and raised C-reactive protein (CRP) $(84.4 \mathrm{mg} / \mathrm{L})$. He had hyponatraemia (serum sodium $119 \mathrm{mmol} / \mathrm{L}$ ) and metabolic acidosis (serum bicarbonate $17 \mathrm{mmol} / \mathrm{L}$ ). His serum creatinine was $5.6 \mathrm{mg} / \mathrm{dl}$ and serum urea was 112 $\mathrm{mg} / \mathrm{dl}$. Chest X-ray revealed left sided pleural effusion, bilateral pulmonary inflammatory lesion with fibrosis and left sided cavitary lesion (Figure 1). His serum lactate dehydrogenase (LDH) (545 U/L), D-dimer (10.36 $\mathrm{mcg} / \mathrm{ml})$ and glycated haemoglobin (HbAlc) (8.3\%) were raised and serum ferritin $(191 \mathrm{ng} / \mathrm{ml})$ and procalcitonin $(0.19 \mathrm{ng} / \mathrm{ml})$ remained normal.

A reverse transcriptase polymerase chain reaction (RTPCR) for severe acute respiratory syndrome coronavirus type 2 (SARS-CoV-2) appeared negative. High resolution computed tomography (HRCT) scan of chest revealed

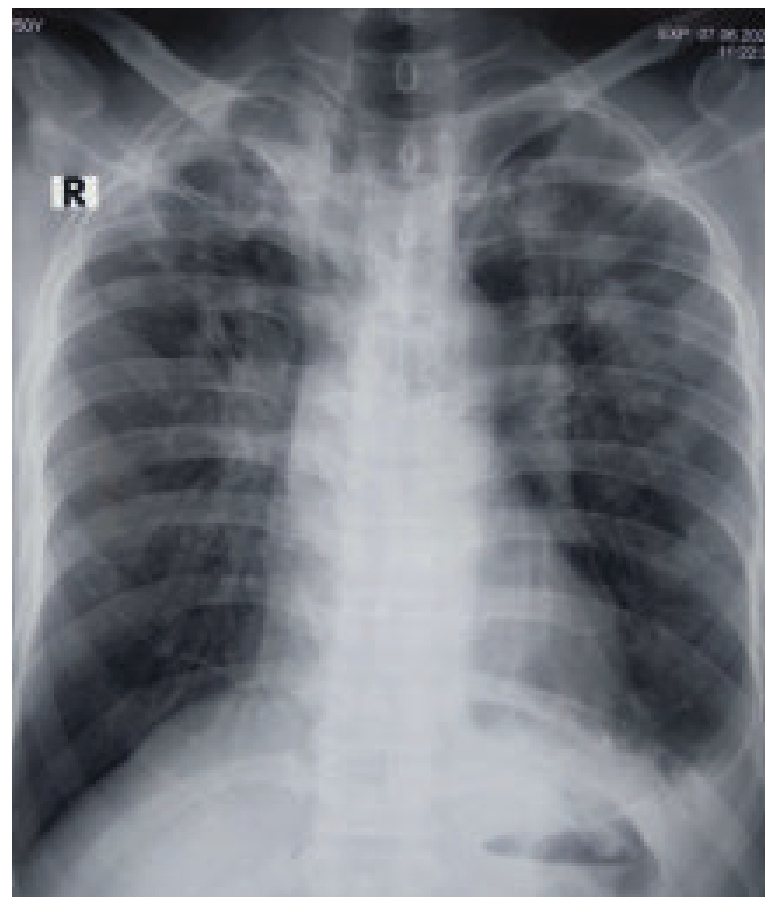

Figure 1 Chest X-ray postero-anterior view showing bilateral lung infiltrates with left sided minimal pleural effusion

bilateral small fibrotic nodules of varying sizes with calcification, bilateral infective bronchiectasis with parenchymal fibrosis and mild upper lobar volume reduction, multiple lung cysts with left sided mild pleural effusion (Figure 2).
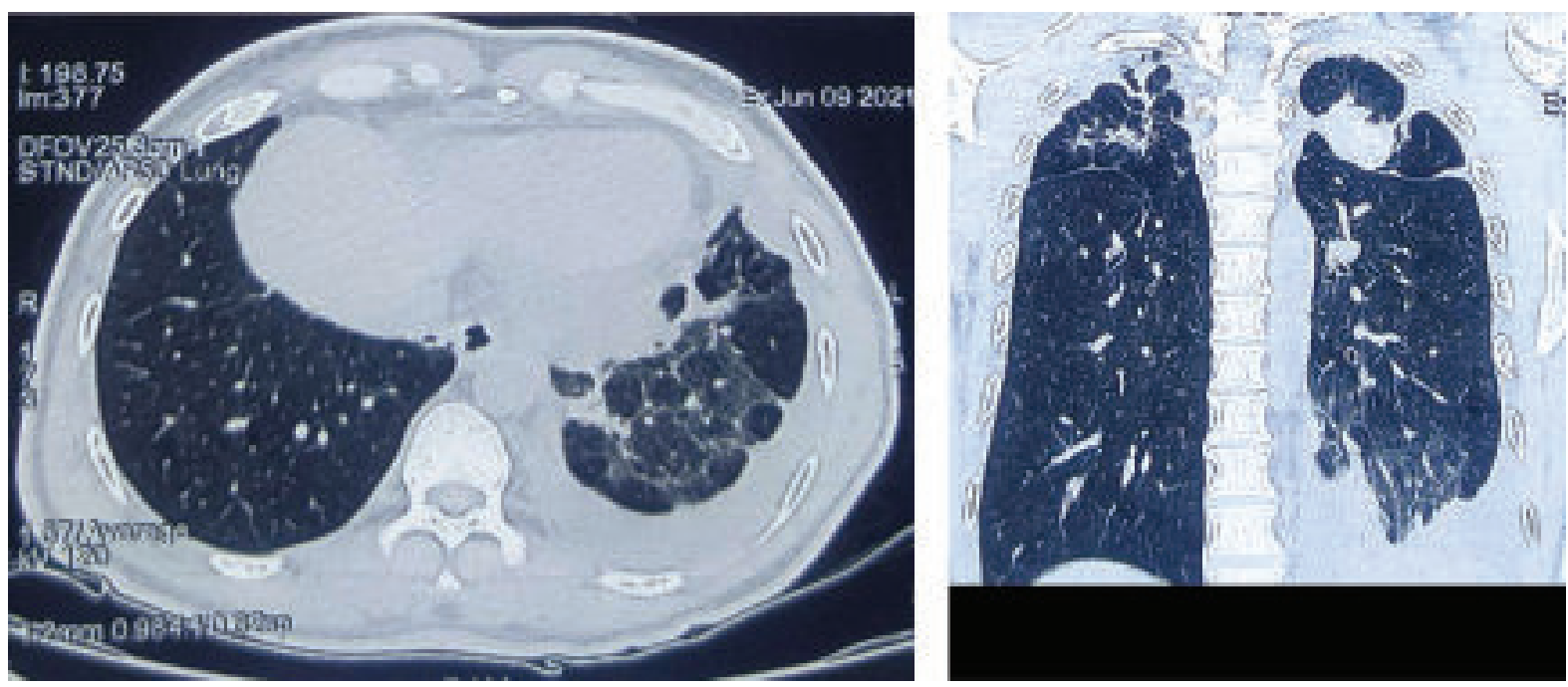

Figure 2 High resolution computed tomography scan of chest (axial and coronal films) showing bilateral small fibrotic nodules with calcification, bilateral infective bronchiectasis with parenchymal fibrosis and mild upper lobar volume reduction, multiple lung cysts and left sided pleural effusion 
Sputum for Gram-staining showed Gram-positive cocci in short and long chains (profuse) and Gram-positive and Gram-negative bacilli (moderate). Direct microscopic examination of sputum revealed plenty of pus cell/high power field (HPF), 10-15 epithelial cell/HPF and characteristics hyphae of Aspergillus (Figure 3) and Mucormycetes fungi (Figure 4). Acid fast bacilli (AFB) were not found and GeneX-pert was negative on sputum examinations. Sputum culture showed moderate growth of Mucor spp. (Figure 5) and Aspergillus spp.

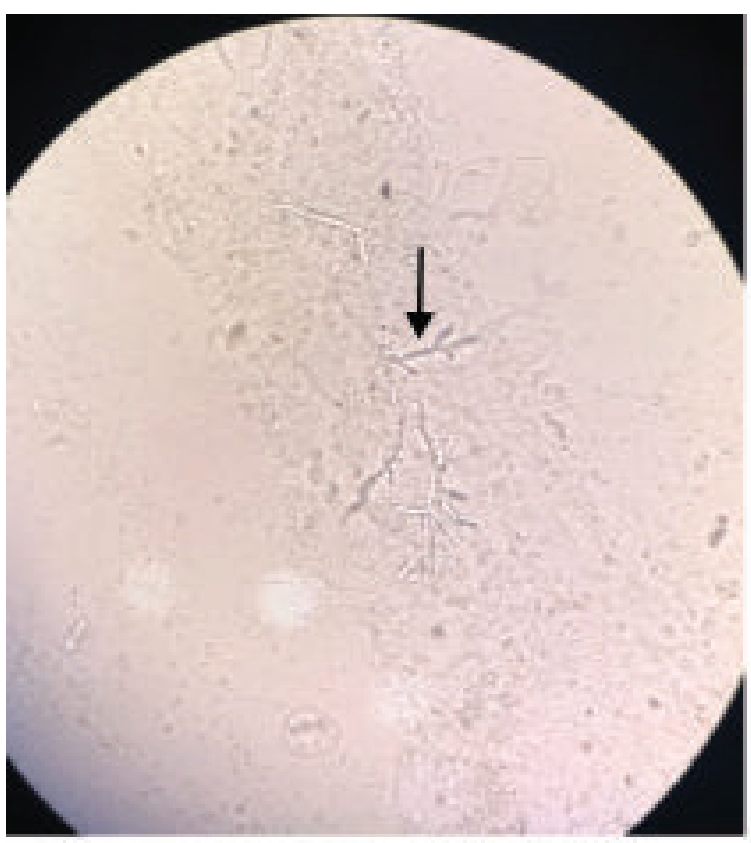

Figure 3 Direct microscopy of sputum showing (10x) of dichotomous branching of Aspergillus hyphae
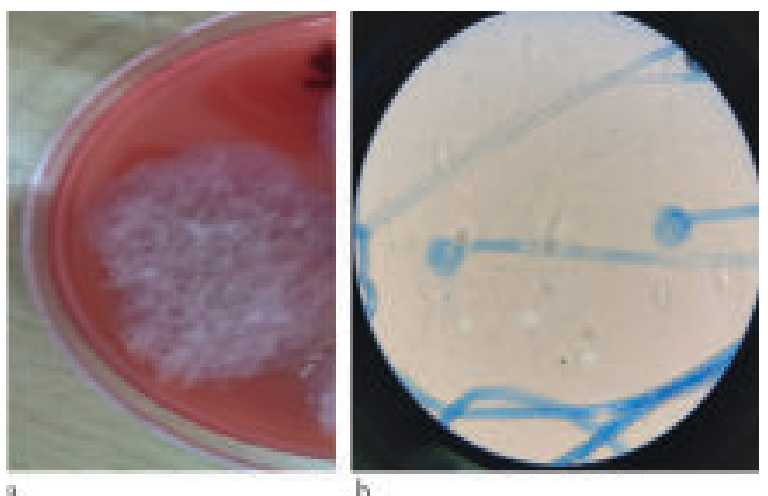

Figure 5 Sputum culture showing (a) growth of Mucor spp. in blood agar and (b) microscopic view from culture plate with lactophenol cotton blue preparation
(Figure 6) with characteristics pattern of spore and conidia with lactophenol cotton blue preparation seen under microscope.

After admission, he was started with ceftazidime (dose adjusted for kidney function) and moxifloxacin (as per local protocol) along with paracetamol and fexofenadine. His anti-diabetic (premixed insulin and linagliptin) and anti-hypertensive medications (cilnidipine and carvedilol) were continued.

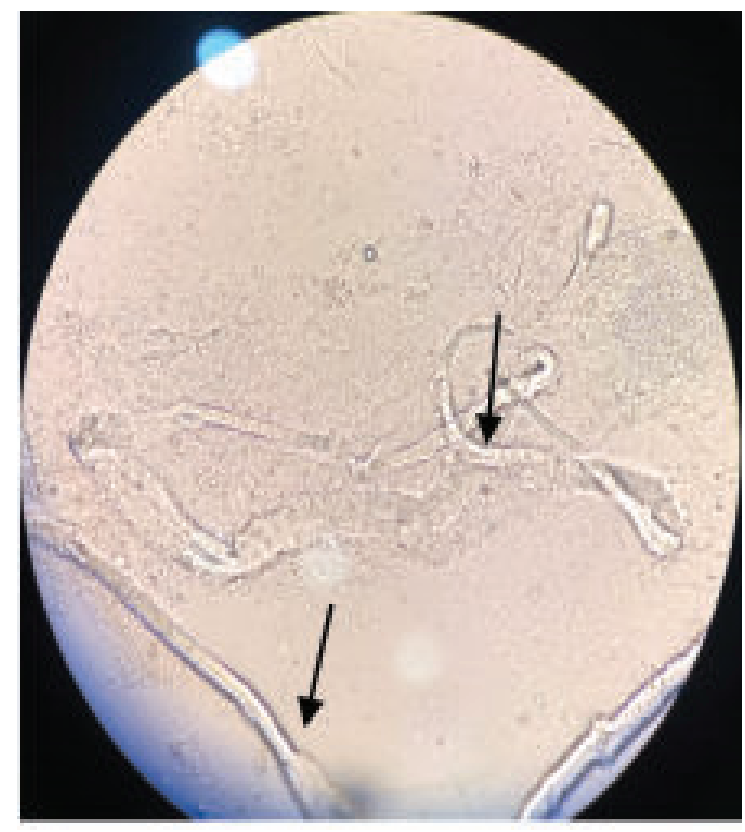

Figure 4 Direct microscopy of sputum showing broad irregular, non-septate hyphae of Mucormycetes
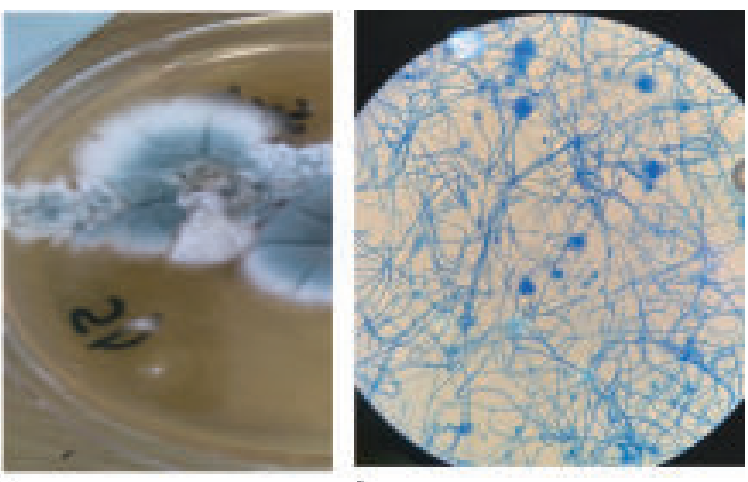

b

Figure 6 Sputum culture showing (a) growth of Aspergillus spp. in Sabouraud dextrose agar (SDA) media and (b) microscopic view from culture plate with lactophenol cotton blue preparation 
His response to antibiotics was satisfactory. He was under close monitoring for possible invasive pulmonary mucormycosis. His steady improvements in clinical, laboratory and imaging parameters helped us deciding not to consider the case as invasive fungal infection, rather these were colonizations in a previously damaged lung. Last follow-up over telephone after eight-weeks of discharge from hospital revealed that he was now free of fever and any respiratory symptoms.

\section{DISCUSSION}

Mucormycosis and other opportunistic infections among patients suffering and recovering from COVID19 have emerged as a challenging health issue throughout the globe, specially more in India. ${ }^{4-6}$ A case of post-COVID pulmonary mucormycosis is reported from Bangladesh ${ }^{7}$ and to our knowledge, few more cases are diagnosed (un-published). Several factors are responsible for such an increased incidence of invasive fungal infections among patients recovering from COVID-19, which include severe pulmonary alveolar damages in COVID-19 that facilitate fungal invasion, immune dysregulations in COVID-19, invasive mechanical ventilations may facilitate entry of opportunistic pathogens, immunosuppressive drugs used in COVID-19, prolonged hospital stay and injudicious antibiotic prescription. ${ }^{7,8}$

Aspergillus spp. are common, ubiquitous and commensals of environment and humans, may survive on surfaces and commonly colonize in human upper airway and lungs, specially in those having chronic respiratory conditions. ${ }^{9}$ Clinical diseases in healthy people are uncommon and there are several definite clinical forms of the disease including invasive pulmonary aspergillosis, chronic necrotizing aspergillosis, allergic bronchopulmonary aspergillosis, aspergilloma, pulmonary aspergillous overlap syndromes etc. ${ }^{9}$ Aspergilloma can occur in cavitary lung lesions like tuberculosis, as was seen in our case and merit no treatment unless produces significant symptoms including haemoptyis.

Mucormycosis is an opportunistic infection, caused by the order Mucorales. ${ }^{10}$ Mucormycosis-causing species include Rhizopus, Mucor, Cunninghamella, Apophysomyces, Lichtheimia (formerly Absidia), Saksenaea, Rhizomucor and others. Mucorales species are ubiquitous saprophytes and soil is believed to be the main habitat of most of these fungi. The sporangiospores released by Mucorales range from 3 to $11 \mathrm{im}$ in diameter and can be aerosolized to disperse in the environment, leading to an air-borne infection in the upper or lower airways. These fungi can invade the nose, sinuses, brain, gastrointestinal tract, skin and lung or even disseminate throughout the body. There are several clinical forms including rhino-orbito-cerebral, pulmonary, cutaneous, gastro-intestinal, genito-urinary and disseminated forms with high mortality except the cutaneous form.

Diagnosis needs high index of clinical suspicion. Microscopy of a representative sterile sample in a clinical suspect is very important, as many cultures are laboratory contaminants. Not only that, cultures may be negative in 40-50 percent of clinical cases. ${ }^{8}$ Many a times, histopathology of biopsy materials (open or endoscopic) or surgically debrided tissues and their cultures may identify the causative agents.

Principles of treatment of invasive fungal infections include early diagnosis, reversal of predisposing factors, if any, surgery in selected cases and antifungals; azoles for aspergillosis and liposomal amphotericin B for mucormycosis, though some oral agents are also of use. These drugs are toxic, have high cost and also are not widely available. Clinical presentation, trends in levels of inflammatory markers, response to treatment, prior respiratory and immunological status of the patient and most importantly combined decision between clinician and microbiologists can guide to determine the status of the patient, management plan and thus the overall outcome.

As Mucorales and Aspergillus are common contaminants of airways, isolation of these organisms from sputum must be interpreted with caution. They may be seen in clinical specimens as a result of oral or naso-gastric feeding or air prior to sample collection. So, growth of a mucormycete in culture of sputum may therefore not represent clinically significant invasive disease as was seen in our cases. However, culture growth of two fungi (Aspergillus and Mucor spp.) in sputum of this post-COVID patient reflected superficial transient colonizations.

Authors' contribution: TAC drafted manuscript. TSUH collected data from the patient and drafted case report. LB was the key microbiologist and revised the 
manuscript. MAR did literature search and revised the manuscript. All authors were involved in management of the patients, read and approved the final manuscript for submission.

\section{Conflicts of interest: Nothing to declare.}

\section{REFERENCES}

1. Chowdhary A, Agarwal K, Meis JF. Filamentous Fungi in Respiratory Infections. What Lies Beyond Aspergillosis and Mucormycosis? PLoS Pathog 2016; 12(4): e1005491.

2. Biswas D, Agarwal S, Sindhwani G, Rawat J. Fungal colonization in patients with chronic respiratory diseases from Himalayan region of India. Ann Clin Microbiol Antimicrob 2010; 9:28.

3. Karan MA, Tunccan OG, Kalkanc A, Arman D, Kustimur S. Widespread Colonization of Mucor circinelloides in a Patient with Type 2 Diabetes and Colon Cancer: Case Report. Turkiye Klinikleri J Case Rep 2014;22(3):18690 .

4. Mahalaxmi I, Jayaramayya K, Venkatesan D, Subramaniam MD, Renu K, Vijayakumar P, et al. Mucormycosis: An opportunistic pathogen during COVID-19. Environmental Research 2021;doi: https://doi.org/10.1016/j.envres. 2021.111643 .
5. Mishra Y, Prashar M, Sharma D, Akash, Kumar VP, Tilak TVSVGK. Diabetes, COVID 19 and mucormycosis: Clinical spectrum and outcome in a tertiary care medical center in Western India, Diabetes \& Metabolic Syndrome: Clinical Research \& Reviews 2021;doi: https://doi.org/10.1016/ j.dsx.2021.102196.

6. Bhogireddy R, Krishnamurthy V, Jabaris SSL, Pullaiah CP, Manohar S. Is Mucormycosis an inevitable complication of Covid-19 in India? Braz J Infect Dis 2021;doi:https:// doi. org/10.1016/j.bjid.2021.101597

7. Afroz F, Barai L, Rahim MA, Kanta SS, Hossain MD. Post-COVID pulmonary mucormycosis: first case report from Bangladesh. Bangladesh J Medicine 2021; 32: 156-60.

8. Khatri A, Chang K-M, Berlinrut I, Wallach F. Mucormycosis after Coronavirus disease 2019 infectionin a heart-transplant recipient - Case report and review of literature. J Med Mycol 2021;31: 101125.

9. Kousha M, Tadi R, Soubani AO. Pulmonary aspergillosis: a clinical review. Eur Respir Rev 2011; 20: 121: 156-74.

10. Muszewska A, Pawºwska J, Krzyœciak P. Biology, systematics, and clinical manifestations of Zygomycota infections. Eur J Clin Microbiol Infect Dis 2014; 33(8): 1273-87. 